DOI: 10.2478/v10122-010-0003-0

\title{
SUR LA TENDANCE ANALYTIQUE DU POLONAIS
}

\author{
MAREK GAWELKO
}

\begin{abstract}
Marek Gawełko. Sur la tendance analytique $d u$ polonais. Lingua Posnaniensis, vol. LII (1)/2010. The Poznań Society for the Advancement of the Arts and Sciences. PLISSN 0079-4740, ISBN 978-83-7654-030-6, pp. 39-54

There are different studies of the analytic tendency in Polish. However, a further study is required to permit the determination of the place occupied by Polish on the evolutive scale of the type «synthetic $\rightarrow$ analytic». The objective of the paper is to establish the distance between Polish and the most evoluated Indo-European languages (English and French) from the point of view of analytism. It is probably true that the passage from synthetic (morphological) structures to analytic (syntactic) ones has taken place sooner in Polish than in Czech. But the two of them are much more synthetic than the most synthetic Romance language (Rumanian) and than German, whose synthetic character, in the domain of Germanic languages, is surpassed only by Icelandic.
\end{abstract}

Marek Gawełko, Włoska 19/20, 30-638 Kraków, Poland

\section{REMARQUES PRÉLIMINAIRES}

Le problème de l'analytisme du polonais a fait l'objet d'une certaine quantité d'études. Le livre de J. Anusiewicz (1978) donne un inventaire assez riche de constructions analytiques. Parmi les raisons de leur création il indique surtout les tendances $1^{\circ}$ à éviter les malentendus, $2^{\circ}$ à la précision, $3^{\circ}$ à introduire des différenciations stylistiques. Il mentionne aussi des raisons extra-linguistiques. Il laisse de côté un problème capital pour l'explication de l'apparition des formes analytiques: le passage des langues indo-européennes de l'état synthétique à l'analytique. Les raisons indiquées par Anusiewicz n'expliquent pas, par ex., l'apparition du pronom sujet en allemand alors que les désinences verbales restent nettes. Comme conséquence de la tendance analytique on devrait indiquer avant tout une restructuration des langues indo-européennes qui entraîne des changements tels que le passage de la ramification de gauche à celle de droite, l'affaiblissement de certaines catégories grammaticales et l'introduction ou le renforcement d'autres. Par ailleurs, la réduction de la typologie au seul facteur morphologique est de vieille date, aujourd'hui plutôt rare.

Les langues romanes ont fait l'objet de maintes classifications. Les classifications morphologiques ou morpho-syntaxiques (par ex. CONTRERAS 1962-1963) sont particulièrement pertinentes sur le plan de l'analytisme/synthétisme d'une langue. Cependant, en dépit du fait que le passage de formes synthétiques aux analytiques se manifeste d'une façon par- 
ticulièrement palpable en morphologie, il conduit à un changement de type de langue. Un tel changement a eu lieu en français (cf. ECKERT 1986). Une langue analytique se distingue d'une langue synthétique à tous les niveaux d'analyse. Pour en rendre compte, il faut choisir des critères variés.

D. Weiss compare le polonais avec le tchèque et le russe. À plusieurs reprises, il constate que le tchèque reste conservateur tandis que le polonais s'achemine vers l'analytisme. Il manque chez lui une perspective plus large. Ainsi, il dit que le tchèque tend à conserver la subordonnée, tandis que le polonais, à introduire une forme non finie (WEISS 1983: 227). On ne sait pas si par ex. l'infinitif polonais a évolué de cette sorte qu'il se rapproche de l'étape finale atteinte dans les langues indo-européennes où il s'avère le plus évolué ou si, au contraire, tout en étant plus fréquent que son équivalent tchèque, il est très éloigné de l'étape évolutive finale de cette forme. Or, la réalité c'est cette dernière éventualité. Ainsi, il est rare comme complément d'adjectif, par ex. fr. elle se sentit heureuse d'être enfermée dans cette aventure d'amour (V 36) mot à mot, 'czuła się szczęśliwa być wmieszana w tę historię miłosną'; il n'apparaît pas après des prépositions, par ex. fr. Souvent j’éprouve un grand ennui à force de lire et d'entendre mot à mot, 'nudzi mnie przez czytać i słuchać'; il ne forme pas de proposition infinitive telle que J'écoute les oiseaux chanter mot à mot, 'słucham ptaków śpiewać', il ne prend pas le sujet au nominatif, par ex. prt. Não tenhas pena de eu não ir contigo. (ML 101) mot à mot, 'nie żałuj, że ja nie iść z tobą'; il est rare en fonction nominale comme dans chcieć to móc 'vouloir c'est pouvoir'. En portugais, il y a même une forme rare dans les langues du monde: infinitif personnel.

En somme, les deux travaux mentionnés, ainsi que tant d'autres concernant la tendance analytique du polonais sont d'une utilité incontestable. Cependant, il n'est pas sans intérêt de situer ce problème dans une perspective indo-européenne. Il s'agirait de dresser une liste de paramètres qui évoluent au cours des millénaires et d'établir, dans le cadre de chaque paramètre, la position du polonais. L'analyse de ces paramètres conduira à établir, d'une façon globale, à quel endroit de la marche vers l'analytisme se trouve le polonais. L'analyse doit donc commencer par l'établissement des paramètres, mais aussi des langues qui seront des points de repère. L'établissement de la liste complète des paramètres ainsi que la prise en compte de toutes les langues indo-européennes sont des procédures impossibles à réaliser.

Il y a une décennie, nous avons traité le même problème (GAWEŁKo 2001a). Cependant, actuellement nous disposons de données sur des fonctions et des critères plus nombreux, d'un corpus plus ample. Les problèmes décrits auparavant seront maintenant limités en principe aux données numériques.

Nous avons choisi comme points de repère l'anglais, les cinq langues romanes officielles (que nous désignons parfois, par souci de simplicité, par le terme: «les langues romanes», de même que le terme «les langues slaves» est employé, chez nous et ailleurs, pour signifier: les langues slaves excepté le bulgare et le macédonien). Ce sont: l'espagnol, le français, l'italien, le portugais et le roumain. Parfois nous faisons appel à l'allemand. Sans compter le persan (cf. Schwegler 1990: 21), l'anglais et le français sont les langues indo-européennes les plus analytiques (cf. GAWEŁKo 2001b). On peut supposer que la grande majorité des paramètres retenus seront à l'étape la plus évoluée au moins dans une de ces langues, par ex. l'étape la plus évoluée de l'infinitif indo-européen est réalisée par le portugais, celle du passif par l'anglais. 


\section{QUELQUES REMARQUES SUR LA MÉTHODE}

La comparaison peut se faire au moins des deux manières: la langue analysée peut être comparée soit statiquement soit dynamiquement. Le principe dynamique de comparaison suppose la marche des langues indo-européennes vers une certaine direction. L'analyse doit rendre compte de la rapidité et de la spécificité de la marche des langues comparées. Nous ne sommes pas isolé à penser que le principe fondamental qui détermine l'évolution de ces langues est la tendance analytique (ex. SAPIR 1921; HARRIS 1978). Nous avons donné des arguments en faveur de cette thèse dans GAWEŁKO (2001b: 27-29).

L'analyse de l'aspect évolutif conduit à dire que les langues slaves, plus synthétiques que les langues romanes, se sont éloignées relativement peu, l'anglais et le français - relativement beaucoup, de la protolangue.

\section{BUT DU TRAVAIL ET CORPUS}

Nous nous assignons la tâche d'établir la force de la tendance analytique du polonais comparée à celle d'un groupe de langues typologiquement différenciées mais toutes plus analytiques que lui sur la base d'un certain nombre de critères. Malheureusement, la prise en compte du latin dans notre étude n'est pas satisfaisante. Néanmoins, certains de ces traits typologiques retenus seront d'une grande utilité à mettre le polonais sur l'échelle indoeuropéenne «synthétique $\rightarrow$ analytique». Les critères appliqués sont assez variés: de nature lexicale, syntaxique et discursive.

Notre corpus embrasse cinq langues romanes: l'espagnol, le français, l'italien, le portugais et le roumain, deux langues germaniques: l'anglais et l'allemand, une langue slave: le polonais. Le latin n'est représenté que par IC.

Le corpus I comporte 400000 mots graphiques environ pour chaque langue. Il se compose de 6 ouvrages dans leur intégrité (4 ouvrages français: $\mathrm{CH}, \mathrm{E}, \mathrm{P}, \mathrm{V}, 1$ ouvrage anglais: A et 1 ouvrage latin: IC), des 3 premiers chapitres d'un ouvrage portugais (ML) et de fragments de deux ouvrages polonais (CD et Q). Pour chaque ouvrage, nous disposons de l'original et de traductions en sept langues (pour IC - en huit langues, y compris le latin). Une exception: le texte CD n'a pas de version portugaise, ce qui nous a forcé à prendre en compte un fragment de la version portugaise d'un autre ouvrage (BA) de longueur équivalente à celle du texte manquant.

Trois de ces ouvrages sont rédigés dans une langue proche de la langue parlée: A, ML et CD. La langue de IC est quelque peu archaïsante.

Autant que c'est possible, nous utilisons ce corpus. Cependant, comme il n'est pas facile de manier une telle masse d'informations, nous avons constitué un autre corpus, défini comme corpus II. Il est beaucoup plus petit et sert à l'analyse de catégories, lexicales et grammaticales, difficiles à formaliser. Il est limité aux quatre romans: $\mathrm{E}, \mathrm{CH}, \mathrm{P}, \mathrm{Q}$. Le nombre de mots varie quelque peu d'une langue à l'autre. Le fragment de chaque roman se limite en principe aux 300 premiers mots comptés dans la version française, à ceci près que nous ne coupons pas les phrases. Comme le corpus doit terminer par un point, il dépasse normalement ce nombre de mots. Ainsi, la version française du corpus comporte $1^{\circ}$ les 29 premières phrases tirées de $\mathrm{E}\left(322\right.$ mots), $2^{\circ}$ les 25 premières phrases de $\mathrm{CH}$ (301 mots), $3^{\circ}$ les 16 premières phrases de $\mathrm{P}(307$ mots $)$ et $4^{\circ}$ les 14 premières phrases de $\mathrm{Q}$ (302 mots). 
Au total, 1232 mots français. Le corpus établi pour les langues restantes, formé des phrases parallèles, varie quelque peu d'une langue à l'autre: 937 mots polonais, 1070 roumains, 1082 allemands, 1099 portugais, 1121 espagnols, 1126 italiens et 1213 anglais.

\section{CRITÈRES LEXICOLOGIQUES}

4.1. Les mots à sémantique pleine prédominent largement dans les langues slaves $(66,3 \%$ en polonais) et d'une façon plus limitée en allemand (56,5\%). Les autres langues prises en compte offrent un équilibre entre les mots de ce type et les mots à fonction grammaticale (cf. GAWEŁKo 2001b: 30).

\subsection{MOTS DÉICTIQUES}

Nous retenons seuls les pronoms personnels et les adjectifs possessifs.

4.2.1. La quantité des pronoms personnels est déterminée avant tout par le caractère obligatoire/facultatif du sujet. Sur la base du corpus I, nous avons établi les occurrences du pronom sujet de la $1^{\text {ère }}$ pers. sg.: fr. je, j'-6243, ang. I-6184, all. ich-6161; prt. eu-1489, esp. yo-1158, it. io - 1035, roum. eu - 722, pol. ja - 631. Pour la $2^{\text {ème }}$ pers. sg., nous indiquons: all. $d u-2009$, it. $t u-717$, roum. $t u-444$ et pol. ty - 305. Étant donné certaines limitations du corpus, en dépit de son ampleur considérable, il est prudent de ne pas différencier les langues qui emploient obligatoirement un sujet grammatical: les données relatives aux trois langues sont trop rapprochées. Cependant, le but principal de ces données est atteint: le polonais est sensiblement plus pauvre en sujets pronominaux que le roumain; à son tour, le roumain est beaucoup plus pauvre à cet égard que n'importe quelle autre langue prise en compte. Le latin et le polonais ne montrent pas de différences; cf. encore IC: lat. ego 130, tu 186; pol.ja 119, ty 175; roum. eu 185, tu 273.

Le critère appliqué ici plaide en faveur d'une distance considérable dans le degré de synthétisme/analytisme entre le polonais et le roumain, et d'une distance très considérable entre le polonais et n'importe quelle autre langue prise en compte.

4.2.2. L'idée de possession est exprimée avant tout par $1^{\circ}$ un verbe de possession, $2^{\circ}$ une construction adnominale telle que l'adjectif possessif ou le génitif et $3^{\circ}$ le datif possessif (cf. CRişTEA 1977: 238 sq). Le datif possessif est relativement fréquent dans les langues synthétiques, comme dans

fr. La sueur coulait sur mes joues. (E 28) - esp. El sudor me corría por las mejillas. - it. Il sudore mi colava sulle guance. - prt. O suor caia-me cara abaixo. - roum. Sudoarea îmi curgea pe obraji. - ang. The sweat was running down my cheeks. - pol. Pot ściekat mi po policzkach. - all. Der Schweiß lief mir über das Gesicht.

Le datif possessif n'apparaît pas dans l'original français. Cependant, il est introduit dans les traductions sauf en anglais. De tels exemples sont relativement fréquents, voir encore:

fr. Quelques gouttes de sueur perlaient sur son front, mais il ne les essuyait pas. (E 28) - esp. Algunas gotas de sudor le perlaban la frente - it. Qualche goccia di sudore gli imperlava la fronte - prt. Algumas gotas de suor escorriam-lhe pela testa - roum. Câteva picături de sudoare $\underline{i} i$ imbroboneau fruntea - ang. A few beads of sweat were forming on his brow - pol. na czole perlito mu się kilka kropli potu - all. Ein paar Schweißtropfen perlten ihm auf der Stirn. 
fr. Toute la nuit, des punaises ont couru sur mon visage. (E 114) - esp. Toda la noche me corrieron las chinches en la cara. - it. Durante tutta la notte mi sono sentito correre le cimici sul viso. - prt. Durante toda a noite, passearam-me piolhos pela cara. - roum. Toată noaptea ploşniţele mi-au umblat pe obraz. - ang. All night I had bugs crawling over my face. - pol. Cała noc pluskwy biegaty mi po twarzy. - all. Während der ganzen Nacht liefen mir Wanzen über das Gesicht.

fr. J'ai essuyé la sueur qui couvrait mon visage (E 137) - esp. el sudor que me cubría el rostro - it. il sudore che mi copriva la faccia - prt. Enxuguei o suor que me cobria a cara - roum. Mi-am șters sudoarea care-mi acoperea faţa - ang. I wiped the sweat from my face - pol. Otarlem pot pokrywajacy mi twarz. - all. Ich wischte mir den Schweiß vom Gesicht.

La différence entre le français et l'anglais d'une part et les autres langues prises en compte de l'autre est évidente. Dans notre corpus II, il y a plus de 20 exemples qui offrent un datif possessif en polonais mais non plus en français. Par contre, les différences entre les autres langues sont limitées et, par conséquent, leur établissement exige l'utilisation d'un corpus considérable. Il paraît que l'allemand s'approche le plus des deux langues et que ce sont le polonais et le roumain qui ont recours au datif pronominal le plus souvent, par ex.

fr. Peut-être à cause des ombres sur son visage, il avait l'air de rire. (E 94) - esp. por el efecto de las sombras sobre el rostro. - it. Forse a causa delle ombre che aveva sul viso - prt. talvez por causa das sombras que se lhe projectavam na cara. - roum. Poate din pricina umbrelor care-i cădeau pe obraz - ang. Perhaps because of the shadows on his face - pol. Prawdopodobnie wskutek cienia, który mu padat na twarz - all. Vielleicht waren die Schatten auf seinem Gesicht schuld daran, daß ich meinte, er lachte.

fr. Il m'a semblé que ses yeux brillaient et que ses lèvres tremblaient. (E 142) - esp. Me pareció que le brillaban los ojos y le temblaban los labios. - it. Mi è sembrato che $i$ suoi occhi fossero lucidi e che le labbra gli tremassero. - prt. Parecia-me que tinha os olhos brilhantes e os lábios trémulos. - roum. Mi s-a părut că ochii îi erau plini de lacrimi şi că buzele $\underline{\underline{i} i}$ tremurau. - ang. I thought I could see his eyes glistening and his lips trembling. - pol. Wydało mi się, że oczy mu się zaszklity $i$ wargi drżały. - all. Es erschien mir, als ob seine Augen feucht schimmerten, und seine Lippen zitterten.

Le datif possessif est possible aussi en français. Par contre, le texte examiné ne note pas d'exemple anglais, par ex.

fr. Je lui caressais les seins. (E 35) - esp. Yo le acariciaba los senos. - it. Io le carezzavo i seni. - prt. Acariciava-lhe os seios. - roum. İ mîngîiam sînii. - ang. and I was fondling her breasts. - pol. Pieścitem jej piersi. - all. Ich streichelte ihre Brüste. La forme polonaise jej n'est pas claire.

fr. les derniers voiles d'eau qui me coulaient dans la bouche. (E 82) - esp. los últimos velos de agua que me corrían hacia la boca. - it. gli ultimi veli d'acqua che mi colavano in bocca. - prt. os últimos véus de água que me escorriam para a boca. - roum. ultimele pinze de apă care mi se scurgeau in gură. - ang. the last few layers of water which trickled down into my mouth. - pol. ostatnie welony wody, która ściekata mi do ust. - all. die letzte Nässe, die mir in den Mund floß.

fr. Mon avocat est venu vers moi, m'a serré la main (E 131) - esp. me estrechó la mano - it. mi ha stretto la mano - prt. apertou-me a mão - roum. mi-a strîns mîna - ang. shook hands - all. drückte mir die Hand - pol. uścisnąt mi rękę.

fr. Tout cela [...] me troublait le regard et les idées. (E 29) - pol. wszystko razem - zmaciło mi wzrok i myśli.

On peut dire que l'adjectif possessif favorise davantage le transitif et, par conséquent, l'énoncé catégorique, tandis que le datif possessif - l'intransitif et, par conséquent, l'énoncé thétique. Ainsi dans fr. Il a perdu son oncle - ang. He lost his uncle - roum. I-a murit un unchi - pol. Umart mu wuj 'est mort lui ${ }_{\text {dat }}$ oncle', les énoncés français et anglais sont catégoriques et respectent le modèle canonique SVO, tandis que les énoncés roumain et polonais sont thétiques et ne respectent pas ce modèle. Ceux-ci sont caractéristiques des langues synthétiques, relativement peu des langues analytiques. 
Le corpus II comporte 28 adjectifs possessifs en anglais, 23 en français et en portugais, moins de 20 dans les autres langues, ce qui revient à dire que l'anglais s'avère le plus analytique.

\subsection{TENDANCE NOMINALE}

L'ordre SOV favorise les catégories verbales, l'ordre SVO - les catégories nominales. On peut donner comme exemple l'aspect verbal dans les langues plus archaïques (le grec., le slave) et l'article dans les langues plus évoluées (ex. romanes).

La tendance nominale peut être déterminée par le rapport entre la quantité des substantifs et celle des verbes finis employés dans un texte parallèle. Ce critère s'avère assez adéquat: dans notre corpus II, l'allemand et le polonais présentent moins de 2 substantifs contre 1 verbe, l'anglais et le français - presque 3: en allemand 1,69 substantif contre 1 verbe, en polonais 1,88, en roumain 1,96; en espagnol 1,97, en portugais 2,11 , en italien 2,39 , en français 2,77, en anglais 2,79. Ces données plaident en faveur de la force de la tendance nominale en anglais et en français, c'est-à-dire en faveur de la force de l'analytisme de ces langues. Elles n'infirment pas le synthétisme du polonais.

Une autre manifestation du style nominal est la quantité considérable des adjectifs. Les données numériques témoignent d'une grande prédominance des adverbes sur les adjectifs dans les langues synthétiques ( 91 contre 51 en polonais, 92 contre 52 en allemand et 78 contre 43 en roumain). En français, c'est l'adjectif qui prédomine.

Les deux critères permettent de constater la plus faible tendance nominale en allemand et en polonais, la plus forte en anglais et en français.

En conclusion, le synthétisme très considérable du polonais se trouve confirmé aussi par la faiblesse relative de la tendance nominale même si nos données ne différencient pas le polonais et l'allemand.

\subsection{VERBES AUXILIAIRES}

Dans GaweŁko (2001a: 19), nous avons présenté quelques chiffres indiquant la rareté des verbes auxiliaires en polonais.

\subsection{MOT GÉNÉRIQUE VS MOT SPÉCIFIQUE}

Dans GaweŁko (2001a: 19-20 et 2001b: 31-32) nous avons donné quelques exemples en faveur de la tendance des langues analytiques au mot générique. Le synthétisme du polonais n'est pas infirmé par ce critère.

\subsection{NOMBRE GLOBAL DES MOTS GRAPHIQUES DANS UN TEXTE PARALLÈLE}

Il y a un rapport évident entre le caractère synthétique/analytique d'une langue et les données numériques ci-dessous, obtenues sur la base de notre corpus I à l'aide d'un programme: l'anglais 422665 mots; le français 414571; 1'espagnol 409492, l'allemand 387361 , l'italien 386071 , le roumain 382423 , le portugais 379421 , le polonais 329442 .

Ce critère apporte un argument en faveur $1^{\circ} \mathrm{d}$ 'un grand synthétisme du polonais, $2^{\circ} \mathrm{d}$ 'un 
synthétisme limité de la majorité des langues romanes et de $1^{\prime}$ allemand et $3^{\circ}$ d'un analytisme des deux langues restantes: 1'anglais s'avère un peu plus analytique que le français.

On pourrait réfléchir sur le rapport entre ce critère lexicologique et différents critères grammaticaux. Notre classification des langues romanes (GAWEŁKO 2001b), où deux langues non romanes ont été prises en compte: le polonais et l'anglais, celui-ci étant plus analytique, celui-là plus synthétique que n'importe quelle langue romane, suggère une conclusion qu'une langue peut être plutôt synthétique du point de vue lexicologique mais plutôt analytique du point de vue grammatical. C'est en l'occurrence le cas du portugais. Cependant, le synthétisme du polonais est très considérable de sorte qu'il est confirmé par toutes sortes de critères: lexicologiques, grammaticaux et communicatifs. Ce problème mérite une attention particulière. Une condition préalable serait de prendre en compte une grande quantité de critères et des langues typologiquement différenciées. Même l'orthographe joue un certain rôle vu que l'emploi de l'ordinateur est basé sur le mot graphique (par ex. tch. nemám - pol. nie mam, fr. pour le voir - esp. para verlo).

\subsection{LES PRÉPOSITIONS DANS LES LANGUES ANALYTIQUES ET SYNTHÉTIQUES}

Les prépositions augmentent leur importance dans les langues analytiques vu qu'elles prennent à leur compte des fonctions exprimées, dans les langues synthétiques, par la flexion. Nous procédons à deux types d'enquêtes. La première est limitée aux quatre prépositions les plus fréquentes dont les occurrences sont établies sur la base de notre corpus I. Étant donné un grand écart entre le polonais et les langues romanes, les formes contractées apparaissant dans celles-ci ne sont pas prises en compte (on évite le problème difficile pour l'analyse à l'ordinateur de distinguer entre deux fr. des: $1^{\circ}$ article indéfini, $2^{\circ}$ de $+l e s$ ).

Le français: de 15565, à 7251, en 4202, dans 3571; 1'anglais: to 12183, of 10522, in 6800, for 3496; l'espagnol: de 18642, a 10179, en 9246, por 4104; l'italien di 10814, a 6409, da 2050, in 4750; le roumain: de 20953, in 7611, cu 5306, pe 5207; 1'allemand: zu 5997 (+ zum 444,+ zur 752), in 4905 (+ im 1373), auf 2819, von 2672 (+ vom 304); le polonais: $w 7370$, na 5032, z 5185, do 3940. La différence énorme qui existe entre le polonais et l'allemand d'une part, entre le polonais et les langues restantes de l'autre est en rapport sans doute avec la persistance ou la disparition de la déclinaison.

La deuxième enquête, fondée sur notre corpus II, rend compte de la totalité des prépositions: 96 occurrences en polonais, 104 en allemand, 142 en roumain, 160 en anglais, 164 en portugais, 171 en français. Le polonais s'avère ici le plus synthétique, l'allemand un peu plus analytique, mais les deux peuvent être traités de synthétiques et opposés aux autres langues qui s'avèrent plutôt analytiques, même le roumain.

\section{CRITÈRES SYNTAXIQUES}

\subsection{ORDRE DES MOTS}

5.1.1. Deux aspects au moins sont en rapport avec la tendance analytique: la liberté de l'ordre et le passage de la ramification de gauche à celle de droite. Dans GAwEŁko (2001a) il est question de la liberté de l'ordre, en particulier de la facilité de l'inversion. 
5.1.2. En ce qui concerne le premier problème, il convient de rappeler que dans notre travail sur la place du sujet dans les langues romanes (augmenté de parallélismes avec l'anglais et le polonais), (1996) on peut voir que l'ordre VS est le plus fréquent en polonais, plus rare dans la majorité des langues romanes, beaucoup plus rare en français et, surtout, en anglais.

5.1.3. En ce qui concerne la tendance à la ramification (détermination) de droite, elle n'a pas été prise en compte.

Or, les langues indo-européennes manifestent la tendance à l'ordre progressif, c'est-à-dire que la ramification de gauche cède le pas à celle de droite. Il y a sans doute un rapport entre la tendance analytique et l'ordre progressif.

Pour déterminer la force de cette tendance en polonais, il convient de rappeler la situation que $1^{\prime}$ on trouve $1^{\circ}$ en latin et $2^{\circ}$ dans les langues prises ici en compte. Or, l'ordre de base en latin classique est $\mathrm{OV}$, même si les débuts du passage de $\mathrm{OV}$ à $\mathrm{VO}$ remontent à l'époque préclassique (cf. Adams 1976: 98; RAmat 1984: 137; PinKster 1988: 255, 281). Dans les langues prises en compte, l'ordre VO, progressif, prédomine largement, à ceci près que le polonais tolère l'ordre SOV si les conditions communicatives sont remplies, au contraire des autres langues où des phrases telles que *Marie la maison vend ne sont plus acceptables.

L'ordre OVS n'est pas très rare dans les langues à déclinaison. L'exemple ci-dessous montre, dans l'original portugais, l'ordre OSV, qui est maintenu dans la majorité des langues romanes, tandis qu'en roumain, en polonais et en allemand, il est remplacé par l'ordre OVS (les deux ne sont pas progressifs):

prt. Quantas oraçöes e sacrifícios ela aí ofereceu ao nosso bom Deus! (ML 35) - fr. Combien de prières et de sacrifices Jacinthe a offerts en ce lieu au bon Dieu! - pol. Ile modlitw i ofiar zlożyło to dziecko tam naszemu dobremu Bogu! - esp. iCuántas oraciones y sacrificios ofreció ella allí a nuestro buen Dios! - it. Quante preghiere e sacrifici ella offrì al nostro buon Dio, in quella grotta! - roum. Câte rugăciuni către Bunul Dumnezeu şi câte jertfe n-a adus în locul acela Jacinta! - ang. How many were the prayers and sacrifices that Jacinta offered there to our dear Lord! - all. Wieviele Gebete und Opfer hat Jacinta dort unserem guten Gott angeboten! La version latine manque; l'ordre OVS y pourrait apparaître.

En somme, toutes les langues, le latin classique excepté, ont un ordre progressif. Les vestiges du vieil ordre OV sont les plus fréquents en polonais.

5.1.4. Place de l'adverbe. En latin, pratiquement seule l'antéposition par rapport au verbe apparaît, les exceptions étant sporadiques dans notre corpus. La situation change en polonais dans ce sens que l'antéposition prédomine largement, mais la postposition n'est pas exceptionnelle. En anglais l'antéposition et la postposition ne sont pas très loin de présenter un équillibre de sorte que le choix du corpus peut donner la priorité à l'un ou à l'autre cas. Par ex.:

lat. Beatus qui horam mortis suae semper ante oculos habet: et ad moriendum cotidie se disponit. (IC) - fr. Heureux celui à qui l'heure de sa mort est toujours présente - prt. Feliz aquele que tem sempre a hora da sua morte diante dos olhos - esp. Dichoso el que tiene siempre como pendiente ante sus ojos la hora de la muerte - it. Beato colui che ha sempre dinanzi agli occhi l'ora della sua morte - roum. Fericit cel ce are totdeauna în faţa ochilor ceasul morţii sale şi zilnic se pregăteşte de moarte. - ang. Blessed is the man who keeps the hour of his death always in mind, and daily prepares himself to die. - pol. Szczęśliwy, kto zawsze ma przed oczami godzinę śmierci i codziennie przygotowuje się do umierania.

Les données numériques ci-dessous sont tirées de notre corpus I. Elles se limitent aux 
adverbes suivants: łatwo, późno, jeszcze, powoli, szybko (fr. encore, facilement, lentement, tard, vite; ang. easily, late, rarely, slowly, still; it. ancora, facilmente, lentamente, raramente, tardi; roum. incet, încă, rar, târziu, uşor). Le but de ce tableau se limite à montrer à quelle étape du passage de la ramification de gauche à celle de droite se trouvent le polonais et quelques langues à forte tendance analytique. On n'a pas pris en compte les adverbes de phrase, mis en tête de la phrase et séparés par une virgule. Les occurrences de ang. still et de roum. încă ont été limitées aux trente premières apparitions, chacun dépassant 200 emplois et affectant démesurément le résultat final.

Tableau 1

\begin{tabular}{|l|c|c|c|c|c|}
\hline $\begin{array}{l}\text { Position } \\
\text { de l'adverbe }\end{array}$ & pol. & it. & ang. & fr. & roum. \\
\hline $\begin{array}{l}\text { Postposition } \\
\text { Antéposition }\end{array}$ & 138 & 122 & 98 & 106 & 176 \\
& 176 & 26 & 71 & 22 & 14 \\
\hline
\end{tabular}

Dans les langues romanes, la postposition est déjà prédominante d'une façon très considérable. Le tableau confirme aussi la conclusion connue au moins depuis PoHL (1965) sur le roumain: c'est la seule langue romane centrifuge, c'est-à-dire que l'ordre progressif y montre une prépondérance nette. C'est un fait paradoxal: contrairement à la tendance générale, le roumain, langue romane la plus synthétique, montre la tendance à la détermination de droite la plus forte. Le paradoxe s'explique par le fait que les langues romanes subissent une influence latinisante, influence relativement faible en roumain. Ainsi dans celui-ci la tendance populaire à la détermination de droite a pu se manifester plus librement que c'était le cas dans les langues sœurs.

La position de l'anglais peut étonner: plus analytique que n'importe quelle langue romane, il s'achemine vers la ramification de droite plus lentement que ces langues. Le problème dépasse la place de l'adverbe. À l'occasion on peut signaler le génitif anglais du type John's book: il précède le nom déterminé, tandis que le génitif en latin classique suivait déjà le nom dans la grande majorité des cas.

Les conclusions sont analogues dans le cas de la place de l'adverbe par rapport à l'infinitif, par ex.

lat. Bene semper agere et modicum de se tenere: humilis animae indicium est. (IC) - pol. Dobrze zawsze czynić $i$ skromnie o sobie mniemać - to znak duszy pokornej. - fr. Faire toujours bien, et s'estimer peu - prt. Proceder sempre bem e ter-se em pouco - it. Agire bene [...].

La différence entre le polonais et toutes les langues prises en compte est très nette. On pourrait dire que la position du polonais se trouve entre le latin et ces langues.

5.1.5 Place de l'adjectif épithète. BAUER constate (1992: 300) que «le nouvel ordre ['ramification de droite'] s'est imposé moins vite dans le syntagme verbal [...] que dans le groupe nominal». L'ordre AN devrait donc être archaïque, tandis que NA - évolué. L'utilité de ce critère est contestable: il conduit, par exemple, à accorder le caractère synthétique à l'anglais et analytique au roumain. Nous le retenons car il est adéquat pour la majorité des langues. 
Nous avons choisi sept adjectifs de qualification pour établir la place qu'ils occupent par rapport au substantif dans notre corpus I: duży, dziki, interesujacy, liczny, ogromny, stary, szczęśliwy (fr. grand, sauvage, intéressant, nombreux, énorme, vieux, heureux; esp. grande, salvaje, interesante, numeroso, enorme, viejo, feliz; roum. mare, sălbatic, interesant, numeros, enorm, vechi, fericit). Ainsi, les langues germaniques ont été écartées vu que l'antéposition de leur adjectif souffre de peu d'exceptions. Pour points de repère nous avons retenu le français, langue romane la plus analytique, le roumain - langue la plus synthétique et l'espagnol comme représentant des langues romanes «du centre». Les exemples sont limités aux trente premiers si la situation est tout à fait claire.

Tableau 2

\begin{tabular}{|c|c|c|c|c|c|c|c|c|}
\hline & \multicolumn{2}{|c|}{ pol. } & \multicolumn{2}{|c|}{ fr. } & \multicolumn{2}{|c|}{ esp. } & \multicolumn{2}{|c|}{ roum. } \\
\hline Adjectif & A & $\mathrm{P}$ & A & $\mathrm{P}$ & A & $\mathrm{P}$ & A & $\mathrm{P}$ \\
\hline duży & 30 & 0 & 30 & 0 & 30 & 2 & 9 & 21 \\
\hline dziki & 17 & 0 & 0 & 10 & 1 & 12 & 0 & 20 \\
\hline interesujący & 2 & 0 & 1 & 5 & 2 & 5 & 0 & 8 \\
\hline liczny & 30 & 0 & 14 & 4 & 10 & 3 & 14 & 8 \\
\hline ogromny & 30 & 0 & 9 & 6 & 27 & 10 & 2 & 9 \\
\hline stary & 30 & 0 & 30 & 0 & 33 & 4 & 16 & 14 \\
\hline szczęśliwy & 18 & 4 & 8 & 16 & 6 & 11 & 10 & 25 \\
\hline
\end{tabular}

$\mathrm{A}$ - antéposition, $\mathrm{P}$ - postposition

Il ressort du tableau que, dans le domaine des adjectifs qui désignent une qualité, le passage de la ramification de gauche à celle de droite est à peine entamé en polonais. Il est le plus avancé en roumain où la postposition prédomine trés largement. Les autres langues romanes présentent une situation intermédiaire (le tableau n'est pas destiné à la décrire).

Il n'en va pas de même si l'adjectif exprime une relation plutôt qu'une qualité graduée, c'est à dire qu'il ne peut former le comparatif et le superlatif, ni être déterminé par des adverbes tels que bardzo, niebywale, etc. (fr. très, énormément), cf. Człowiek bardzo szczęśliwy, mais *Polska bardzo Ludowa, fr. Un homme très heureux, mais *La Pologne très Populaire; *przemyst bardzo chemiczny, *l'industrie très chimique.

En comparant la rapidité du passage de la ramification de gauche à celle de droite dans les deux domaines: la place de l'adverbe et celle de l'adjectif, nous pouvons constater, sur la base des deux tableaux, que l'opinion de Bauer rappelée ci-dessus n'est pas confirmée par notre matériel: la postposition de l'adverbe est antérieure à celle de l'adjectif. Cependant, à en croire P. RAMAT (1984: 141), en latin classique l'ordre progressif prédominait déjà dans le groupe nominal (Nom + Génitif et Nom + Adjectif).

\subsection{ENCORE SUR L'ORDRE DES MOTS EN LATIN ET EN POLONAIS}

Certains ordres, fréquents dans les vieilles langues indo-européennes, deviennent rares en latin classique, mais cessent d'être usuels en polonais. Il s'agit de l'ordre GEN/N (Génitif + Nom), rare en latin (1 exemple sur quatre dans notre corpus, ex. Vanitas est carnis deside- 
ria sequi - IC). Il en va de même du tour «verbe modal + infinitif» (ex. Fac nunc quod tunc facere velles - IC). Le latin classique emploie sporadiquement l'ordre «proposition relative + nom» (cf. AdAMs 1976: 86-87) ainsi que des postpositions (opposées aux prépositions), formes qui n'apparaissent plus dans les langues slaves.

En somme, le latin s'est éloigné moins des vieilles langues, le polonais - davantage, mais les deux langues acceptent en principe les vieux ordres (sauf l'antéposition de la relative et les postpositions en polonais), ce qui n'est plus le cas dans les autres langues prises en compte.

\subsection{QUELQUES CONSTRUCTIONS QUI PRÉDOMINENT DANS LES LANGUES DU TYPE OV ET CELLES PROPRES À L'ORDRE VO}

Il s'agit de constructions $1^{\circ}$ qui augmentent (le passif en être, l'infinitif) ou $2^{\circ}$ qui diminuent (l'impersonnel) leur vitalité avec le progrès de l'analytisme.

Les langues dépourvues de déclinaison ne peuvent pas thématiser le patient en conservant purement et simplement la phrase active. Elles ont recours à la construction passive du type être + participe passé ou à une autre construction telle que la dislocation. Ces constructions sont donc fréquentes dans les langues analytiques, plus rares dans les synthétiques. Dans notre travail (GAwEŁKo 1999: 14), nous offrons les fréquences suivantes du passif en être, basées sur un corpus comportant 170000 mots environ pour chaque langue: 1'anglais 1301 , l'allemand 938, le français 785 , le roumain 776 , l'italien 671 , le portugais 626 , l'espagnol 514 et le polonais 426. La conclusion générale que le passif de la langue la plus analytique est le plus riche et celui de la langue la plus synthétique - le plus faible ne peut pas surprendre. On voit cependant que les différenciations typologiques secondaires ne déterminent pas la vitalité du passif, ce qui s'explique d'une façon relativement simple. Le rôle du passif est double: thématisation du patient et dégradation de la fonction d'agent. Or, ces fonctions sont obtenues aussi par le recours à d'autres constructions. Par exemple, la faiblesse considérable du passif espagnol en ser, estar est en rapport avec la richesse de son passif pronominal (cf. GAWEŁKO 1998-1999: 117).

Comme le passif en être, l'infinitif augmente aussi sa vitalité avec le progrès de l'analytisme. Sur la base d'un corpus comportant 45000 mots environ, nous avons obtenu les données suivantes (GAWEŁKo 2005b: 138-140): le portugais 1512 occurrences, l'espagnol 1433, l'italien 1431, le français 1404, l'anglais 1105, l'allemand 1018, le polonais 903 et le roumain 172. La faiblesse de l'infinitif roumain est un trait balkanique. Le rapport entre la richesse de l'infinitif et l'analytisme de la langue où il est employé n'est pas très étroit. Néanmoins, le roumain excepté pour la raison signalée, le polonais, langue la plus synthétique, a l'infinitif le plus faible.

Contrairement à ces deux constructions (passif en être et infinitif), l'impersonnel, comme le verbe intransitif en général, s'affaiblit avec le progrès de l'analytisme (cf. BAUER 2000: 97). Dans GaweŁko (2008: 79-80) nous présentons les données fondées sur un corpus constitué de 15400 mots environ: 22 verbes impersonnels polonais (75 occurrences), 14 verbes français (53 occurrences), 14 it. (47), 14 all. (38), 12 roum. (53), 8 port. (38) et 2 ang. (26). À l'exception du français et, jusqu'à un certain point, du roumain, ces données montrent un rapport avec la tendance analytique: l'impersonnel polonais est le plus riche, vu que la langue est la plus synthétique, tandis que l'impersonnel anglais, donc celui de la langue la plus analytique, est le plus faible. 


\section{CRITÈRES COMMUNICATIFS}

\subsection{LA PFP (PERSPECTIVE FONCTIONNELLE DE LA PHRASE) ET LE SYNTHÉTISME DU POLONAIS}

Un problème important ce sont les exigences de la PFP: l'énoncé devrait commencer par un thème (ce dont on parle dans l'énoncé; information donnée), celui-ci devrait être suivi par un rhème (ce qu'on dit sur le thème; information nouvelle). C'est à l'école de Prague que revient le principal mérite de décrire ce problème. Dans toutes sortes de théories il y a des éléments faux. Ici aussi il y a une conception fausse qui est généralement acceptée sans être suffisamment prouvée. Telle est la thèse qui dit - en prenant le potentiel pour le réel - que les langues synthétiques se conforment bien aux exigences de la PFP, tandis que les langues analytiques, où l'ordre des mots est chargé de fonctions grammaticales, se conforment mal à ces exigences (nous avons présenté le problème plusieurs fois, dans GAWEŁKO 2001a: 15 il a été signalé, mais seulement en 2003 présenté en détail). Nous nous limitons maintenant à rappeler la conclusion principale: en réalité, ce sont les langues analytiques qui se conforment mieux aux exigences de la PFP que ne le font les langues synthétiques. L'explication en est dans le fait que la liberté de l'ordre de celles-ci sert dans une large mesure à satisfaire les exigences stylistiques et rythmiques.

Étant donné ce principe, nous avons établi, sur la base de deux enquêtes (2003), que le polonais se conforme à un degré moindre aux exigences de la PFP que c'est le cas dans les langues romanes et en anglais, ce qui revient à dire qu'il se comporte comme une langue synthétique ou, plus précisément, plus synthétique que ne le sont les langues romanes et l'anglais.

\subsection{L’ÉNONCÉ THÉTIQUE ET LE SYNTHÉTISME DU POLONAIS}

Les énoncés: thétique et catégorique existent à partir de la protolangue. L'énoncé thétique a un caractère général, il répond à une question, explicite ou implicite, du type: Qu'estce qu'il y a? ou Qu'est-ce qui se passe?, tandis que l'énoncé catégorique prend pour point de départ un détail de la situation d'énonciation générale pour former un énoncé binaire (élément donné + élément nouveau). La question type est Que fait le thème? Au cours des millénaires, l'énoncé thétique a diminué son importance, ce qui se manifeste surtout par la diminution de la fréquence de la proposition impersonnelle. Nous avons vu au $\S 5.3$ que l'impersonnel polonais est plus fréquent que ses équivalents romans ou anglais (cf. encore GAWEŁKo 2005a: 26-27). Il apporte donc un argument en faveur du synthétisme du polonais.

Le comportement de l'impersonnel trouve son équivalent sur le plan communicatif. Il s'agit surtout de l'importance de l'énoncé thétique. Dans les langues analytiques, ce type d'énoncés se limite pratiquement aux verbes existentiels, en polonais les verbes non existentiels sont toujours acceptables. En polonais, à la question générale du genre Qu'est-ce qui se passa alors? on peut répondre: Rozlegt się śmiech, tandis que la réaction normale en français peut être: Il y eut un rire.

L'action de la phrase ci-dessous se passe dans un logement: fr. La sonnette de la porte retentit. (P 106) - pol. Rozległ się dzwonek przy drzwiach. Le français apporte la phrase 
du type SV ou, en termes communicatifs, un énoncé catégorique, le polonais - un énoncé thétique.

L'énoncé thétique - plus fréquent dans les vieilles langues, synthétiques bien entendu, vu qu'il était formé aussi en recourant à des verbes non existentiels - persiste dans une large mesure en polonais, ce qui apporte un argument de plus en faveur de son synthétisme.

\section{REMARQUES FINALES}

En règle générale, la classification d'un groupe de langues indo-européennes est fondée sur un certain nombre de paramètres. Une langue peut recevoir une étiquette en vertu de son comportement dans le cadre de la majorité des paramètres. Pour donner un exemple, il convient de choisir les langues romanes qui ont fait l'objet de maintes classifications. Ainsi le roumain peut être considéré comme la langue romane la plus synthétique vu qu'il est la seule langue romane qui ait conservé les vestiges de l'ancienne déclinaison des substantifs, qu'il jouit de la plus grande liberté de l'ordre des mots, qu'il est la seule langue romane dépourvue de présentatif du type il y a, qu'il abandonne le sujet plus souvent que les langues sœurs, que son impersonnel est le plus riche, etc. Cependant, en dépit de cette étiquette (langue la plus synthétique), bien méritée d'ailleurs, il présente des traits propres par excellence aux langues analytiques. Par exemple, il est considéré, au moins à partir de Pohl, comme la langue où, pour employer des termes à la mode de nos jours, l'ordre des mots est le plus progressif (c'est-à-dire où la ramification de droite est la plus avancée). Il est aussi la seule langue romane qui ait élaboré un indice d'infinitif analytique (a) correspondant à ang. to ou à all. $z u$. Il a aussi un passif fort en être, etc.

Nous rappelons ces quelques faits dans une présentation de l'analytisme du polonais et pour cause. Peu importe qu'il soit opposé au roumain, langue romane la plus synthétique, ou à n'importe quelle autre langue romane, il sera plus synthétique dans la totalité ou, pour être plus prudent, dans la quasi-totalité des critères utilisés. Cette prudence vient de l'impossibilité de prévoir tous les paramètres possibles. Ajoutons le «critère de synthèse» de GrEENBERG (1960; morphèmes divisés par mots) qui, appliqué à un fragment de IC, a donné les résultats suivants: lat. 1.77, pol. 1.67, it. 1.45, ang. 1.15.

En dépit de cas spéciaux, une conclusion est sûre: le polonais s'avère plus synthétique au moins dans la quasi-totalité des critères retenus non seulement comparé avec les langues indo-européennes les plus analytiques (l'anglais et le français) mais aussi avec n'importe quelle autre langue romane.

La situation ne devrait pas être différente si pour point de repère nous choisissons les langues germaniques. Dans cette optique, il serait utile de comparer le polonais avec l'islandais qui est le plus conservateur. Notre corpus nous permet de faire quelques juxtapositions avec l'allemand qui, au point de vue du conservatisme, occupe la deuxième place sur la liste des langues germaniques (cf. éd. GuChMAn 1977: 145, 347; KubrJaKova 1970: 161 passim). Il n'est pas sans intérêt de rappeler ce que dit GrEENBERG (1974: 32) à propos de cette langue: "German is a typical synthetic language (if synthesis is defined in terms of the morpheme-word ratio)». Cependant, comme nous basons notre définition sur des critères variés, notre opinion n'est pas aussi univoque. 
Or, contrairement au polonais, l'allemand a un certain nombre de traits typiques pour les langues analytiques. Il a des articles, un présentatif (es gibt), un indice d'infinitif ( $z u$ ), un passif fort, un infinitif fréquent (nominalisé au moyen d'un article, il est plus fort que c'est le cas dans n'importe quelle langue romane), un aspect verbal extrêmement faible. La déclinaison, qui donne l'impression d'une langue synthétique, y est faible, notamment dans ce sens que l'opposition fondamentale, celle entre le nominatif et l'accusatif, est réduite aux seuls noms masculins singuliers.

D. Weiss a sans doute raison de dire à plusieurs reprises, en analysant différentes catégories grammaticales et lexicales, que le polonais s'avère plus analytique que le tchèque. Cependant, cela ne devrait pas conduire à penser que, dans la marche des langues indoeuropéennes vers l'analytisme, il se caractérise par un progrès considérable. Il est même beaucoup plus synthétique que la langue romane la plus synthétique.

\section{BIBLIOGRAPHIE}

Adams James N. 1976. «A typological approach to Latin word order». Indogermanische Forschungen 86, 70-99.

ANUSIEWIcz Janusz. 1978. Konstrukcje analityczne we współczesnym języku polskim. Wrocław: Ossolineum.

BAUER Brigitte L.M. 1992. Du latin au français: le passage d'une langue SOV à une langue SVO. Nijmegen: Univ. diss.

Bauer Brigitte L.M. 2000. Archaic Syntax in Indo-European. Berlin et New York: Mouton de Gruten.

BRANG Peter, Nivat Georges, Zett Robert (eds.). 1983. Contributions des savants suisses au IXe congrès international des slavistes à Kiev, septembre 1983. Bern: Peter Lang.

Contreras Heles. 1962-1963. «Una clasificación morfo-sintáctica de las lenguas románicas». Romance Philo$\log y 16,261-268$.

CRIŞTEA Teodora. 1977. Éléments de grammaire contrastive. Domaine franço-roumain. Bucarest: Editura Didactică şi Pedagogică.

ECKERT Gabriele. 1986. Sprachtypus und Sprachgeschichte. Untersuchungen zum typologischen Wandel des Französischen. TBL 265. Tübingen: Narr.

GawetKo Marek. 1996 et 1999. L'étude sur l'ordre des mots dans les langues romanes. Vol. 1: La position du sujet; vol. 2: La passivation. Lublin: Towarzystwo Naukowe.

GAWEŁKo Marek. 1998-1999. «Le passif réfléchi roman». Roczniki Humanistyczne XLVI-XLVII, 5, 115-143.

GAwEŁKo Marek. 2001a. «Język polski wobec tendencji analitycznej języków indoeuropejskich». Polonica 21, $11-24$.

GaWEŁKo Marek. 2001b. «Essai de classification fonctionnelle des langues romanes». Romance Philology 55, $21-40$.

GAwEŁKo Marek. 2003. «Zasady perspektywy funkcjonalnej zdania a tendencja analityczna języków». Poradnik Językowy 3, 22-32.

GAWEŁKO Marek. 2005a. «La perspective indo-européenne de l'impersonnel polonais». Wiener Slavistisches Jahrbuch 51, 21-30.

GaweŁKo Marek. 2005b. «Sur la vitalité de l'infinitif roman». Alfa. Revista de Lingüística (Brésil) 49, 2, $133-151$.

GaweŁKo Marek. 2008. «Sur le développement de l'impersonnel indo-européen». Lingua Posnaniensis 50, 175-193.

GreENBERG Joseph. 1960. «A Quantitative Approach to the Morphological Typology of Language». International Journal of American Linguistics 26, 178-194.

GreenBerg Joseph. 1974. Language Typology. A Historical and Analytic Overview. The Hague-Paris: Mouton.

Guchman Mira M. (éd.) 1977. Istorico-tipologičeskaja morfologija germanskich jazykov. Moscou: Izdatel'stvo Nauka.

HARRIS Martin. 1978. The Evolution of French Syntax. London: Longman. 
KUBRJAKOva Elena S. 1970. «Morfologičeskaja struktura slova v sovremennyx germanskix jazykach». In: SEREBRENNIKOV 1970.

PINKSTER Harm. 1988. Lateinische Syntax und Semantik. Tübingen: Francke Verlag.

PoHL Jacques. 1965. «Le roumain, seule langue romane centrifuge?». In: Omagiu lui Alexandru Rosetti la 70 de ani. Bucarest: Ed. Acad. Republicii Socialiste România, 709-717.

Ramat Paolo. 1984. Linguistica tipologica. Bologna: Il Mulino.

SAPIR Edward. 1921. Language. New York: Harcourt.

Schwegler Armin. 1990. Analycity and Syntheticity. A Diachronic Perspective with Special Reference to Romance Languages. Berlin, New York: Mouton de Gruyter.

Serebrennikov Boris A. (ed.). 1970. Obšče jazykoznanie. Formy suščestvovanija funkcii, istorija jazyka. Moskva: Nauka.

WeIss Daniel. 1983. «Zur typologischen Stellung des Polnischen (ein Vergleich mit dem Čechischen und Russischen)». In: BRANG et al. 1983: 219-245.

\section{CORPUS}

$1^{\circ}$ Camus, A. L'étranger, Paris, Gallimard, 1942; El extranjero, Madrid, El Libro de Bolsillo, 1982; Lo straniero, Milan, Tascabili Bompiani, 1988; O estrangeiro, Lisbonne, Unibolso, s.d.; Străinul, in Străinul, Ciuma, Căderea, Exilul şi împărătia, Bucarest, RAO International Publishing Company, p. 27-92, 1993; The Outsider, Londres, Penguin Books, 1983; Der Fremde, Reinbeck bei Hambourg, Rowohlt, 1993; Obcy, Varsovie, Krąg, 1991. (E)

$2^{\circ}$ Camus, A. La chute, Paris, Gallimard, 1989; La caida, Madrid, Alianza Editorial, 1982; La caduta, Milan, Tascabili Bompiani, 1989; A Queda, Lisbonne, Livros do Brasil e Editorial Verbo, 1971; Căderea, in Străinul, Ciuma, Căderea, Exilul şi împărăţia, Bucarest, RAO International Publishing Company, p. 295-369, 1993; The Fall, Londres, Penguin Books, 1963; Der Fall, Reinbeck bei Hambourg, Rowohlt, 1995; Upadek, Varsovie, Krąg, 1991. (CH)

$3^{\circ}$ Camus, A. La peste, Paris, Gallimard, 1989; A peste, Lisbonne, Livros do Brasil, s.d.; La peste, Barcelone, Edhasa, 1977; La peste, Milan, Tascabili Bompiani, 1989; Ciuma, in Străinul, Ciuma, Căderea, Exilul şi împărăţia, Bucarest, RAO International Publishing Company, p. 93-294, 1993; The plague, Londres, Penguin Books, 1960; Die Pest, Hambourg, Rowohlt, 1994; Dżuma, Varsovie, Jota, 1991. (P)

$4^{\circ}$ Maupassant, G. de. Une vie, Paris, Gallimard, 1974; Una vida, in G. de Maupassant, Obras completas, p. 149-285, Madrid, Aguilar, 1965; Uma vida, Mem Martins, Livros de bolso Europa-America, 1974; Una vita, Farigliano, Arnoldo Mondadori Editore, 1984; O viaţă, Chişinău, Făt-Frumos, 1994; A Woman’s Life, Londres, Penguin Books, 1965; Ein Leben, Frankfurt/M-Berlin, Ullstein, 1995; Historia jednego życia, Varsovie, Zrzeszenie Księgarstwa, 1985. (V)

5 Memórias da Irmã Lúcia, Fátima, Postulação, 1978; Mémoires de Sœeur Lucie, Fátima, Postulação, 1980; Memorias de la Hermana Lucía, Fátima, Postulação, 1978; Memorie di Suor Lucia, Fátima, Postulação, 1980; Sora Lucia despre Fatima, Fátima, Secretariado dos Pastorinhos, 2002; Fatima in Lucia's own words, Fátima, Postulação, 1976; Schwester Lucia spricht über Fátima, Fátima, 1975; Siostra Łucja mówi o Fatimie, Fátima, Postulação, 1978 (jusqu'à la p. 104 de l'original portugais). (ML)

$6^{\circ}$ Tomas a Kempis, De imitatione Christi; ( $O$ naśladowaniu Jezusa Chrystusa), édition bilingue, Varsovie, Instytut Wydawniczy Pax, 1981; L'imitation de Jésus-Christ, Paris, Édition du Seuil, 1961; L'imitazione di Cristo, Milan, Edizioni Paoline, 1988; Imitación de Cristo, Barcelone, Editorial Regina, 1987; Imitação de Cristo, Braga, Editorial Franciscana, 1980; Urmarea lui Hristos, Timişoara, Editura Metropoliei Banatului, 1991; The Imitation of Christ, Londres, Penguin Books, 1965; Thomas von Kempen, Die Nachfolge Christi, Kevelaer, Butzon \& Bercker, 1990; Tomasz à Kempis, Naśladowanie Chrystusa, Cracovie, WAM, 2000. (IC)

$7^{\circ}$ Carrol, L. Alice's adventures in Wonderland - Les aventures d'Alice au Pays des merveilles, Paris, AubierFlammarion, 1970; Aventuras de Alicia en el país de las maravillas, Barcelone, Moby Dick, 1973; Alice nel Paese delle Meraviglie, in Alice nel Paese delle Meraviglie, Attraverso lo specchio, Milan, Garzanti, p. 1-141, 1989; Alice no País das Maravilhas, Mem Martins, Publicações Europa-America, 1998; Alisa în ţara minunilor, Craiova, Editura Literatorul, 1991; Alice im Wunderland, Hambourg, Cecilie Dressler Verlag, 1990; Przygody Alicji w Krainie Czarów, Varsovie, Lettrex, 1990. (A) 
$8^{\circ}$ Sienkiewicz, H. Quo Vadis, Varsovie, Labos, 1990; Quo vadis, Paris, Le livre de poche 3161, 1971; Quo vadis? Madrid, Aguilar, 1967; Quo vadis?, Milan, Biblioteca Universale Rizzoli, 1984; Quo Vadis?, Mem Martins, Livros de bolso Europa-America, 1974; Quo Vadis, Bucarest, Editura Universul, 1945; Quo Vadis?, Sawtry-New York, Hippocrene-Dedalus, 1993; Quo Vadis, Zurich, Diogenes, 1985 (chap. X-XII du premier volume). (Q)

$9^{\circ}$ Andrzejewski, J. Popiót i diament, Varsovie, Krajowa Agencja Wydawnicza, 1966; Cendres et diamant, Paris, Gallimard, 1967; Cenizas y diamantes, Barcelone, Luis de Caralt, 1966; Cenere e diamanti, Lerici Editori, Milan, 1961; Cenuşă şi diamant, Bucarest, Editura pentru Literatură Universală, 1968; Ashes and Diamond, Eveston, Northwestern Univ. Press, 1991; Asche und Diamant, Frankfurt/Main, Suhrkampf Verlag, 1984 (chap. IV et $\mathrm{V})(\mathrm{CD})$

$10^{\circ}$ Maupassant, G. de. Bel-ami, Livros de bolso Europa-América, Guimarães Editores, 1972. (BA)

Allatum die 23 mensis Juni anno 2009 